\title{
Bilateral Pulmonary Agenesis: A Rare and Unexpected Finding in a Newborn
}

\author{
Lananh N. Nguyen, MD ${ }^{1}$ W. Tony Parks, MD² \\ ${ }^{1}$ Department of Pathology, Dartmouth-Hitchcock Medical Center, \\ Lebanon, New Hampshire \\ 2 Departments of Pathology and Obstetrics, Gynecology and \\ Reproductive Sciences, Magee Women's Hospital, University of \\ Pittsburgh School of Medicine, Pittsburgh, Pennsylvania
}

\begin{abstract}
Address for correspondence W. Tony Parks, MD, Departments of Pathology and Obstetrics, Gynecology and Reproductive Sciences, Magee Women's Hospital, University of Pittsburgh School of Medicine, 300 Halket Street, Pittsburgh, PA 15213 (e-mail: parkswa@upmc.edu).
\end{abstract}

Am J Perinatol Rep 2016;6:e246-e249.

\begin{abstract}
Keywords

- bilateral pulmonary agenesis

- lung anomaly

- developmental anomaly

Background Bilateral pulmonary agenesis is a rare congenital anomaly incompatible with life that can be missed on routine prenatal screening. Prenatal ultrasound diagnosis of this fatal anomaly can aid in prenatal counseling and postdelivery care.

Case Study We report the case of a newborn who was born prematurely at 29 weeks gestation and underwent several unsuccessful intubation attempts immediately after delivery. Conclusion Autopsy examination revealed bilateral pulmonary agenesis with a short, blindly ending trachea.
\end{abstract}

Developmental lung anomalies encompass a wide spectrum of pulmonary aberrations. Abnormalities of pulmonary parenchymal size/growth represent a subset of developmental pulmonary anomalies. In pulmonary hypoplasia, the mildest form, the lungs develop incompletely but nearly normally. The overall architecture is roughly normal, but the lungs are small and the airspace branching is limited. This entity can present clinically in the perinatal period or be identified at autopsy using lung weight criteria, radial alveolar counts, or a lung weight:body weight ratio. Pulmonary aplasia is a more severe form, with significantly less lung development. This anomaly is specifically defined as an absence of lung parenchyma in the presence of bronchial buds. The most severe abnormality of pulmonary parenchymal size/growth is pulmonary agenesis. This lesion is defined by the absence of three components: the lung parenchyma, bronchus, and pulmonary vasculature. ${ }^{1}$ All of these lung anomalies can affect one lobe or multiple lobes, in addition to presenting as varying combinations of hypoplasia and aplasia. Within this spectrum of pulmonary developmental anomalies, pulmonary agenesis is seen in the lowest frequency. When pulmonary agenesis does occur it is commonly unilateral, with the failure of either the right or left pulmo- nary components to develop. True bilateral pulmonary agenesis has been described only rarely in the literature relative to the thousands of reported cases of unilateral pulmonary agenesis, bilateral pulmonary hypoplasia/aplasia and unilateral pulmonary hypoplasia/aplasia. This report presents the case of a newborn with unsuspected bilateral pulmonary agenesis.

\section{Case Report}

The newborn was born at 29 4/7 gestational weeks to a 21year old G1P0 female with a past medical history significant for bipolar disorder and depression. No family history of congenital anomalies was elicited. The mother's medication history was unremarkable history. Prenatal screening for hepatitis B, HIV, syphilis, and Rubella was negative. By maternal report, an ultrasound anatomy scan performed at an outside institution was interpreted as unremarkable with no evidence of polyhydramnios. On the day of delivery, the mother presented to an outside hospital for preterm labor that progressed despite tocolysis. The newborn was delivered via cesarean delivery for a transverse lie. At delivery, the heart rate was normal but the newborn was apneic with no cry. received

March 29, 2016

accepted after revision

May 6, 2016
DOI http://dx.doi.org/

10.1055/s-0036-1584530. ISSN 2157-6998.
Copyright $\odot 2016$ by Thieme Medical

Publishers, Inc., 333 Seventh Avenue, New York, NY 10001, USA. Tel: +1(212) 584-4662.
License terms

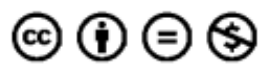



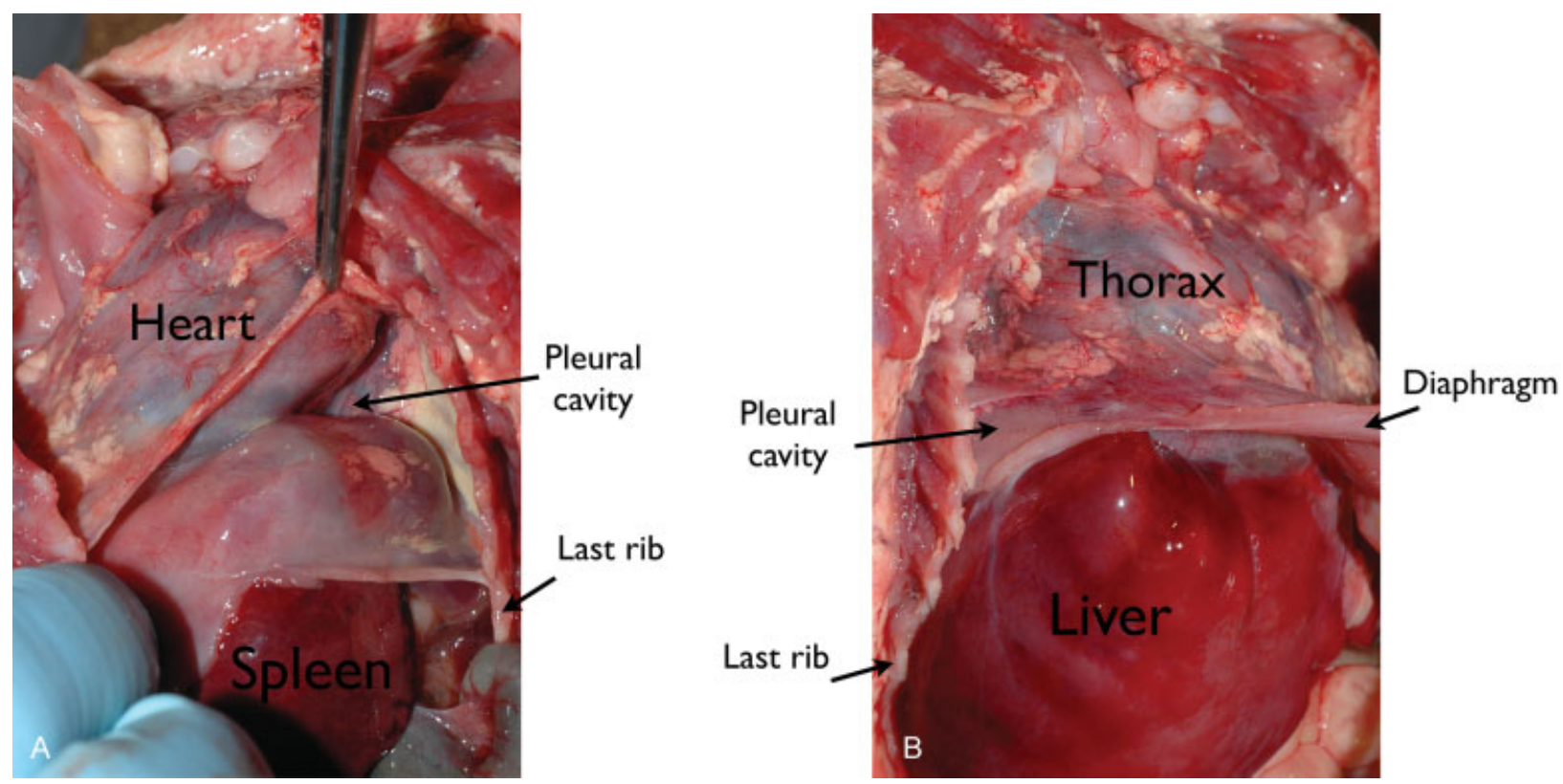

Fig. 1 Internal anatomy showing high diaphragm and absence of pleural contents. There was complete absence of lung parenchyma or bronchial components in both the left or right pleural cavities. (A) Left lateral view showing empty pleural cavities resulting in a cephalad displacement, of the diaphragm and spleen. (B) Right lateral view showing empty pleural cavities resulting in cephalad displacement of the diaphragm and liver.

Resuscitation was begun, including chest compressions and bag-mask ventilation. Endotracheal intubation was unsuccessful despite multiple attempts by several team members. Each reported that the endotracheal tube met resistance at a point just beyond the visible vocal cords. The patient's oxygen saturation decreased rapidly and the newborn expired 26 minutes after delivery.

\section{Autopsy}

At autopsy, body weights and measurements of the newborn were consistent with gestational age. Cytogenetic studies showed a normal female 46,XX karyotype.

On external examination, the newborn showed no craniofacial, abdominal, or genitourinary abnormalities. The chest appeared normal in size and shape. A single 1-mm, pedunculated, dark red-tan probable hemangioma at the nipple line in the midanterior chest was noted.

On internal examination, there was a high diaphragm and small a thoracic space that was occupied almost exclusively by the heart. The most striking finding was noted upon opening the left and right pleural spaces. The pleural cavities were nearly empty bilaterally, devoid of recognizable pulmonary contents, lung parenchyma, or anomalous pulmonary segments ( - Fig. 1). In the neck, the trachea measured approximately $1 \mathrm{~cm}$ in length and narrowed to a blind end distally as identified by a firm cartilaginous nodule (-Fig. 2 ). The anterior neck structures, including the larynx, vocal cords, thyroid, and cervical thymus, appeared unremarkable.

The heart showed a complete endocardial cushion defect. Two vessels originated from the left atrium with paths similar to the superior and inferior vena cava. The aortic arch was mostly normal except for an anomalous right subclavian artery distal to its regular branches. The pulmonary trunk originated in the right ventricle and terminated in the aorta as the patent ductus arteriosus. No pulmonary arteries were present; however, dimples on the interior surface of the trunk marked their possible incomplete origins. The superior and inferior vena cava were unremarkable.

The gastrointestinal contents were malrotated. The ileum was present in the left middle to lower quadrant, and the remainder of the small bowel was located on the right side. The colon ascended from the rectum but then looped to the right lower abdomen, then across the abdomen into the left lower abdomen, and the remainder of the colon was present in the left quadrants. The appendix was located on the left lower quadrant. The

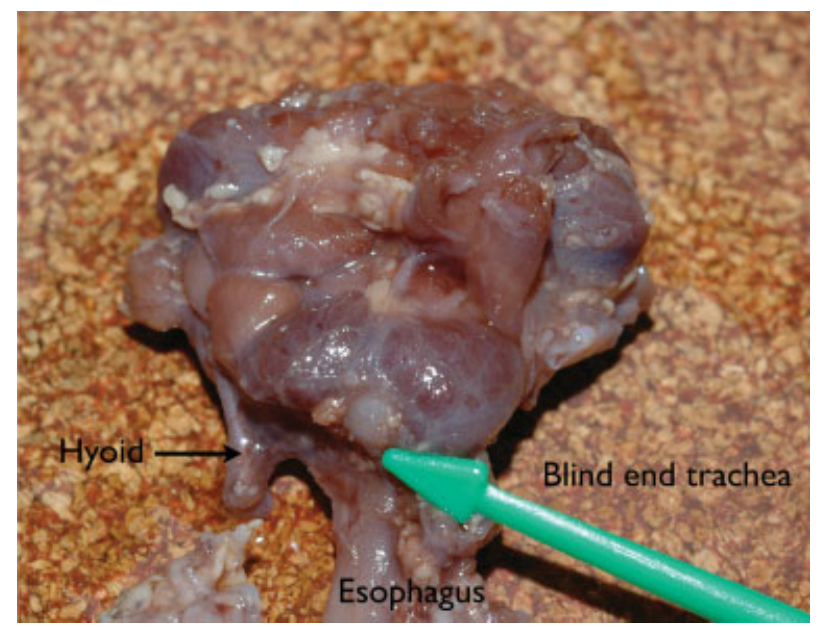

Fig. 2 Neck contents demonstrating termination of the trachea. The green arrow points to the blindly ending trachea that extended $1 \mathrm{~cm}$ below the vocal cords. 
remainder of the viscera was unremarkable, including esophagus, stomach, appendix, liver, spleen, pancreas, kidneys, ureters, bladder, adrenals, uterus, and cervix. The ovaries were unremarkable with the minor exception of a single 1-mm yellow redtan nodule on the distal portion of the right ovary. A two-vessel umbilical cord was present. The brain was not examined at the request of the patient's family.

Microscopic examination of the neck contents showed progressive narrowing of the tracheal airway distally, with focal calcifications of the cartilaginous tracheal rings. Fibroadipose tissue with some lymphoid and neural components was noted outside of the parietal pleura. There was no architectural or cellular component resembling pulmonary or bronchial components identified. No significant microscopic findings were present in the remaining organs.

\section{Discussion}

We have presented a rare case of bilateral pulmonary agenesis in a newborn with an apparently normal ultrasound anatomy scan delivered after preterm, premature rupture of membranes. Moreover, this current case of bilateral pulmonary agenesis has few developmental anomalies and a normal karyotype, and it may thus be unrelated to current known genetic defects, syndromes, or associations.

During normal lung development, the lower respiratory system forms at approximately 4 weeks when the foregut produces an outpouching in the ventral wall. This outpouching, the primordial lung bud, then forms the trachea and bifurcates to form the left and right lung buds. Mesodermal tissue surrounds the lung buds and ultimately forms the cartilaginous, muscular, and connective tissues of the trachea and lung. As the lung buds and their bronchi grow caudally, they also will differentiate and ramify to form the bronchioles and alveoli, which are sufficiently matured for life at 7 months but continue to mature for years after birth. ${ }^{2}$ The fourth week of development is also the time when the atrioventricular endocardial cushions of the heart begin to develop. Since the timing of the development of the two primary anomalies in this case-aberrant early tracheal/lung development and defective endocardial cushion formationshows considerable temporal overlap, it seems likely that a localized disruptive event occurred at that time to affect these two adjacent organ systems. The etiology for this disruptive event, whether mediated through a genetic, syndromic, vascular or other mechanism, is not clear.

Although there is currently no known mutational defect, syndrome, or association similar to this presented case, we did consider similar developmental conditions. Matthew-Wood syndrome-also known as Spear syndrome ${ }^{3}$; pulmonary agenesis, microphthalmia, and diaphragmatic defect (PMD) syndrome ${ }^{4}$; or pulmonary hypoplasia/agenesis, diaphragmatic hernia/eventration, anophthalmia/microphthalmia, and cardiac defect (PDAC) syndrome ${ }^{5}$-was first identified in a fetus with bilateral pulmonary agenesis and anophthalmia. ${ }^{6}$ Since then, these syndromes, which all have characteristic eye pathology, have been associated with mutation in the stimulated by retinoic acid 6 gene (STRA6). ${ }^{7}$ In our patient, the eyes were anatomically normal, making these entities less likely in the differential diagnosis. Hydrolethalus syndrome, Ellis-van Creveld syndrome, Opitz G/BBB syndrome, Smith-Lemli-Opitz syndrome, Meckel syndrome, C syndrome, Fryn syndrome, and Goldenhar syndrome are other syndromes that all have been described with abnormal lung development. However, these syndromes have characteristic facial or other external anomalies that were not present in this case. Tracheal agenesis association, VACTERL (vertebral anomalies, anal atresia, cardiac malformations, trachea-esophageal fistula, renal dysplasia, and limb abnormalities) association, and Mardini-Nyhan association are conditions that have tracheal abnormalities, but all are associated with skeletal defects that were not identified in our case.

Another surprise in this case was the reportedly normal prenatal ultrasound anatomy scan. Since the mother was transferred from an outside institution, the prenatal records were not available and clinical management was solely based on reported history. The bilateral absence of pulmonary development was unknown and unexpected until detected at autopsy examination. Whether this unforeseen outcome could have been prevented is an issue worthy of elaboration. Pulmonary agenesis, particularly when bilateral, may be difficult to detect on imaging. Previous reports have documented missed bilateral pulmonary agenesis ${ }^{8,9}$ on prenatal imaging, where only subtle findings of heart predominance in the thoracic cavity, slight deviation in the cardiac axis, and an elevated diaphragm were seen. Additional imaging modalities such as magnetic resonance imaging, fetal echocardiogram, and Doppler imaging, in addition to reevaluation at a later gestational age, have shown to be effective in demonstrating the lack of lung parenchyma and pulmonary vasculature. $^{9-11}$ In our patient, only one ultrasound anatomy scan was mentioned by the mother; no other imaging studies appear to have been done.

We have presented the case of a newborn who rapidly succumbed to unanticipated bilateral pulmonary agenesis. Although exceedingly rare, this entity must be considered when managing a newborn presenting with unexpected respiratory distress.

\section{Conflict of Interest}

The authors report no conflicts of interest.

\section{References}

1 Graff GR, Beckerman RC. Radiological case of the month. Pulmonary aplasia. Arch Pediatr Adolesc Med 1997;151(7):737-738

2 Sadler TW. Medical Embryology. 13th ed. Philadelphia, PA: Wolters Kluwer Health; 2015:218-224

3 Spear GS, Yetur P, Beyerlein RA. Bilateral pulmonary agenesis and microphthalmia. Am J Med Genet Suppl 1987;3:379-382

4 Berkenstadt M, Lev D, Achiron R, Rosner M, Barkai G. Pulmonary agenesis, microphthalmia, and diaphragmatic defect (PMD): new syndrome or association? Am J Med Genet 1999;86(1):6-8

5 Chitayat D, Sroka H, Keating S, et al. The PDAC syndrome (pulmonary hypoplasia/agenesis, diaphragmatic hernia/eventration, anophthalmia/microphthalmia, and cardiac defect) (Spear syndrome, Matthew-Wood syndrome): report of eight cases including a living child and further evidence for autosomal recessive inheritance. Am J Med Genet A 2007;143A(12):1268-1281 
6 Ostör AG, Stillwell R, Fortune DW. Bilateral pulmonary agenesis. Pathology 1978;10(3):243-248

7 Golzio C, Martinovic-Bouriel J, Thomas S, et al. Matthew-Wood syndrome is caused by truncating mutations in the retinol-binding protein receptor gene STRA6. Am J Hum Genet 2007;80(6): 1179-1187

8 Veluppillai C, Jossic F, Quéré MP, Philippe HJ, Le Vaillant C. Rare case of bilateral pulmonary agenesis and prenatal diagnosis [in French]. Gynecol Obstet Fertil 2014;42(7-8):533-536
9 Lee KA, Cho JY, Lee SM, Jun JK, Kang J, Seo JW. Prenatal diagnosis of bilateral pulmonary agenesis: a case report. Korean J Radiol 2010; 11(1):119-122

10 Ramanah R, Martin A, Guigue V, et al. Recurrent prenatally diagnosed isolated bilateral pulmonary agenesis. Ultrasound Obstet Gynecol 2012;40(6):724-725

11 Vettraino IM, Tawil A, Comstock CH. Bilateral pulmonary agenesis: prenatal sonographic appearance simulates diaphragmatic hernia. J Ultrasound Med 2003;22(7):723-726 